\title{
Original
}

\section{Isolation and Characterization of the Polyhexamethylene Biguanide Hydrochloride-Resistant Fungus, Purpureocillium lilacinum}

\author{
TAKAKO YAMAMOTO', YIKELAMU ALIMU ${ }^{2}$, HIROKI TAKAHASHI ${ }^{2,3,4}$, YOKO KUSUYA ${ }^{2}$, \\ KOUICHI HOSOYA ${ }^{1}$, NAOFUMI SHIGEMUNE ${ }^{1}$, SATOSHI NAGAI ${ }^{1}$, AND TAKASHI YAGUCHI ${ }^{2}$ * \\ ${ }^{1} R \& D$-Safety Science Research, Kao Corporation, 2606 Akabane, Ichikai-machi, Haga-gun, Tochigi 321-3497, Japan \\ ${ }^{2}$ Medical Mycology Research Center, Chiba University, 1-8-1 Inohana, Chuo-ku, Chiba 260-8673, Japan \\ ${ }^{3}$ Molecular Chirality Research Center, Chiba University, 1-33 Yayoi-cho, Inage-ku, Chiba 263-8522, Japan \\ ${ }^{4}$ Plant Molecular Science Center, Chiba University, 1-8-1 Inohana, Chuo-ku, Chiba 260-8673, Japan
}

Received 8 March, 2021/Accepted 22 April, 2021

We isolated a fungus from a $20 \%(=200,000 \mu \mathrm{g} / \mathrm{mL})$ aqueous solution of polyhexamethylene biguanide hydrochloride (PHMB), a widely used antimicrobial and examined its morphology and drug resistance profile. Based on the sequence of the internal transcribed spacer region of ribosomal DNA, the fungus was identified as Purpureocillium lilacinum. Although the $P$. Iilacinum type and resistant strains showed similar morphology, the latter had extremely low PHMB susceptibility and was able to grow in $20 \%$ aqueous solution of PHMB, which eliminated the type strain. The minimum inhibitory concentration (MIC) of PHMB for the resistant strain was significantly higher than that of the type strain and other pathogenic filamentous fungi and yeasts. The susceptibility to antimicrobial agents and antifungal agents other than PHMB was similar to that of the type strain, therefore the drug resistance of the isolate was specific to PHMB. Furthermore, we sequenced the genome of the isolate to predict PHMB resistance-related genes. Despite its high resistance to PHMB, no well-known genes homologous to fungal PHMB-resistant genes were detected in the genome of the resistant strain. In summary, $P$. lilacinum was found to be significantly more resistant to PHMB than previously reported, via an unidentified mechanism of drug resistance.

Key words : Purpureocillium lilacinum / Polyhexamethylene biguanide hydrochloride / Minimum inhibitory concentration / Drug resistance / Genome sequence.

\section{INTRODUCTION}

Antimicrobial agents are widely used to eliminate or suppress the growth of microorganisms. Polyhexamethylene biguanide hydrochloride (PHMB), a broad-spectrum antimicrobial and antiviral agent, is an effective disinfectant and biocide even when used at low concentrations. PHMB is active against both gram-positive (minimal inhibitory concentrations (MIC) of PHMB for Bacillus subtilis ATCC 6633 and methicillinresistant Staphylococcus aureus NCTC 11940 are 5 and

*Corresponding author. Tel: +81-43-226-2790, Fax: +81-43226-2486, E-mail : yaguchi (a)chiba-u.jp
$2.5 \mu \mathrm{g} / \mathrm{mL}$, respectively) and gram-negative bacteria (MIC of PHMB for Escherichia coli ATCC 9001 and Pseudomonas aeruginosa ATCC 25668 are 5 and 310 $\mu \mathrm{g} / \mathrm{mL}$, respectively) (Chadeau et al., 2012). PHMB is also active against yeasts $\left(\mathrm{MIC}_{90}\right.$ in Candida albicans isolates associated with infective keratitis is $1.56 \mu \mathrm{g} / \mathrm{mL}$ ) (Messick et al., 1999), filamentous fungi (a $5 \mu \mathrm{g} / \mathrm{mL}$ PHMB treatment significantly inhibited arthroconidia germination and mycelial growth of Geotricum citri-aurantii) (Feng et al., 2011), and viruses (PHMB was effective in vitro against herpes simplex virus type 1 at concentrations as low as 0.01\%) (Valluri et al., 1997). Additionally, PHMB is water-soluble, odorless, colorless, thermostable, and $\mathrm{pH}$-stable and presents low toxicity to mammals. 
Therefore, it is globally used in food, industrial, domestic, and institutional settings for its antimicrobial properties (Thomson et al., 1981; Mashat, 2016; Hubner and Kramer, 2010). According to the manufacturer's recommendation, 20-200 and 200-400 $\mathrm{mg} / \mathrm{mL}$ PHMB should be used for water treatment applications and the disinfection of pre-cleaned solid surfaces, respectively (Arch Chemicals, Inc., Norwalk, CT, USA, 2005).

It is important to select an antimicrobial agent based on its antimicrobial spectrum and evaluate the concentration, $\mathrm{pH}$, contact time, and temperature suitable for use. Inappropriate and excessive use of antimicrobial agents has led to concerns regarding microbial contamination in various industries and the emergence of drug-resistant variants of clinically relevant pathogens. Makela et al. (1991) reported that the lowest concentrations of commercial germicides recommended by manufacturers are not sufficient to eliminate the slime-producing lactic acid bacteria in rooms and on the equipment of meat-processing plants. Henly et al. (2019) demonstrated that long-term exposure of uropathogenic $E$. coli to commonly used biocides lowers its susceptibility to these agents, which may be explained by phenotypic alterations impacting biofilm formation, antibiotic susceptibility, and relative pathogenicity. Thus, the emergence of antimicrobial resistance and contamination by resistant microorganisms is a critical issue.

It has been proposed that PHMB adheres to the cell membrane and mainly exerts its antimicrobial activity by disrupting the membrane structure in prokaryotic cells. Recently, Allen et al. (2006) demonstrated that a direct and strong cooperative binding between PHMB and genomic DNA leads to bacterial cell death, presenting a new theory for the mechanism of action of PHMB. However, this theory does not apply to eukaryotic cells that have more extensive compartmentalization of genomic DNA, in which internal structures act as barriers blocking the direct interaction between PHMB and DNA. Elsztein et al. (2011) examined the cellular mechanisms responsible for low susceptibility in the PHMB resistant Saccharomyces cerevisiae strain JP-1, and provided biological evidence supporting the participation of NCW2, which encodes the novel cell wall protein 2, in cell tolerance to PHMB (Elsztein et al., 2016). NCW2 was found to be among the cell wall integrity genes of S. cerevisiae, and had an auxiliary function in the maintenance of the glucan/chitin balance and cell wall structure (Queiroz et al., 2020).

By serendipity, we noted white floating masses in a $20 \%(=200,000 \mu \mathrm{g} / \mathrm{mL})$ aqueous stock solution of PHMB. The mass exhibited mycelial morphology under a microscope; subsequently, we isolated a fungus from the contaminated PHMB solution. This study aimed to investigate the microbiological characteristics of the isolated PHMB-resistant fungus and its resistance profile against major antimicrobial agents to understand the clinical implications and public health concerns associated with such resistant fungi. Furthermore, we sequenced the genome of the isolate to elucidate its genomic features and predict PHMB resistance-related genes, in reference to the previously reported NCW2 gene sequence. Our findings may be applied in the future to prevent the emergence of PHMB-resistant fungi.

\section{MATERIALS AND METHODS}

\section{PHMB aqueous solution}

The PHMB aqueous solution used for fungal detection was obtained by repackaging a sample from Vantocil ${ }^{\mathrm{TM}}$ IB (Lonza, Basel, Switzerland), a 20\% aqueous solution of $\mathrm{PHMB}$, aliquoted into $20-\mathrm{L}$ polyethylene containers, and was stored statically at $20-25^{\circ} \mathrm{C}$ for more than 1 month.

Each concentration of PHMB aqueous solution for susceptibility testing was prepared by diluting Vantocil ${ }^{\mathrm{TM}}$ IB with sterile ion-exchanged water.

\section{P. lilacinum strains and culture conditions}

P. lilacinum IFM $47467^{\top}\left(\mathrm{NBRC} 5350^{\top}\right)$, NBRC 5752, NBRC 31847, NBRC 31914, NBRC 31970, NBRC 32861, and NBRC 103232 were obtained from NITE Biological Resource Center (Tokyo, Japan), and Aspergillus fumigatus IFM 54229 (= Af293), A. niger IFM 62238, Fusarium solani IFM 62065, Penicillium citrinum IFM 62178, C. albicans IFM 40009, and Cryptococcus neoformans IFM 46660 were obtained from the Medical Mycology Research Center (Chiba University, Chiba, Japan) through the National Bio-Resource Project of the MEXT, Japan (TABLE 1). The stock cultures were revived under the conditions recommended by the respective culture collections. The working cultures were maintained on potato dextrose agar (PDA) slants at $4{ }^{\circ} \mathrm{C}$. The strains were cultivated on PDA plates at $22.5-25^{\circ} \mathrm{C}$ until sporulating colonies of appropriate size were observed.

\section{Fungal isolation}

White floating masses were collected from a 20\% aqueous solution of $\mathrm{PHMB}$ with a micropipette and were morphologically examined under a light microscope (ECLIPSE Ni-U; Nikon Co., Tokyo, Japan) equipped with a digital camera (DS-Fi2; Nikon Co., Tokyo, Japan). Mycelia were inoculated on PDA plates and incubated at $22.5^{\circ} \mathrm{C}$ for 6 days. Single colonies were re-inoculated three times on new PDA plates to obtain pure isolates. 
TABLE 1. List of culture strains used in this study

\begin{tabular}{lll}
\hline \multicolumn{1}{c}{ Scientific name } & \multicolumn{1}{c}{ Culture No. } & \multicolumn{1}{c}{ Source } \\
\hline Purpureocillium lilacinum & IFM $47467^{\top}\left(\right.$ NBRC 5350 $\left.{ }^{\top}\right)$ & Soil \\
& NBRC 5752 & Unknown \\
& NBRC 31847 & Paddy field soil \\
& NBRC 31914 & Silk-worm food \\
& NBRC 31970 & Soil \\
& NBRC 32861 & Living leaf, Phragmites karka \\
Aspergillus fumigatus & NBRC 103232 & Collembolan, Arthropleona, Collembola \\
Aspergillus niger & IFM 54229 & Clinical specimen \\
Fusarium solani & IFM 62238 & Otorrhea \\
Penicillium citrinum & IFM 62065 & Clinical specimen \\
Candida albicans & IFM 62178 & Scalp \\
Cryptococcus neoformans & IFM 40009 & Patient with pulmonary candidiasis \\
\hline
\end{tabular}

\section{Identification of fungal isolates}

The genomic DNA of the isolates was extracted by physical disruption and by following Marmur's method with slight modifications (Marmur, 1961). The complete nuclear internal transcribed spacer (ITS) region, including ITS1, 5.8S, and ITS2, was PCR-amplified using specific primers ITS5 (White et al., 1990) and NL4 (O'Donnell, 1993) and PrimeSTAR HS DNA Polymerase (Takara Bio Inc., Shiga, Japan). The PCR products were sequenced using a BigDye Terminator Cycle Sequencing Kit v3.1 (Thermo Fisher Scientific Inc., Waltham, MA, USA). The complete nuclear ribosomal ITS regions were sequenced using ITS5 and ITS4 primers (White et al., 1990). The sequences were assembled using ChromasPro 1.7 (Technelysium Pty, Ltd., Tewantin, QLD, Australia). Multiple sequence alignments were performed using CLUSTAL W (Thompson et al., 1994), and the final sequence alignments were manually edited to remove ambiguous positions and gaps. Phylogenetic trees were constructed by the neighbor-joining method (Saitou and Nei, 1987) using the Kimura two-parameter model (Kimura, 1980) in MEGA ver.6.0 (Tamura et al., 2013). Branch reliability was assessed using 1000 bootstrap replicates (Felsenstein, 1985). DNA extraction, PCR amplification, DNA sequencing, and molecular phylogenetic analyses were performed at TechnoSuruga Laboratory Co., Ltd. (Shizuoka, Japan).

\section{Scanning electron microscopy (SEM)}

Morphological differences between the fungal isolate and the type strain P. lilacinum IFM $47467^{\top}$ cultivated on PDA plates at $25^{\circ} \mathrm{C}$ for 14 days were evaluated using an
S-3400N scanning electron microscope (Hitachi Ltd., Tokyo, Japan).

\section{PHMB susceptibility testing}

Spore suspensions of $P$. lilacinum strains were prepared from 2-week-old cultures by gently scraping the mycelial surfaces with sterile cutters, suspending the scrapings in $0.05 \%(\mathrm{w} / \mathrm{v})$ Tween-80, and filtering the solutions through sterile gauze to collect the spores. The spores were counted using a hemocytometer, and homogenous suspensions of $10^{6}$ spores $/ \mathrm{mL}$ were prepared. Then, $200 \mu \mathrm{L}$ of $10^{6}$ spores $/ \mathrm{mL}$ suspension was mixed with $20 \mathrm{~mL}$ of fresh $20 \%$ PHMB aqueous solution and incubated at $25^{\circ} \mathrm{C}$. At $3,7,14$, and 28 days, the numbers of live spores were determined by diluting the suspensions in lecithin and polysorbate 80 solutions (Nihon Pharmaceutical Co., LTD, Tokyo, Japan) as neutralizers and spreading on PDA plates. The plates were incubated at $22.5^{\circ} \mathrm{C}$ for 6 days, and colony-forming units (CFU) were counted. The detection limit was $2.0 \log \mathrm{CFU} / \mathrm{mL}$.

\section{MIC testing}

The spore suspensions of $P$. lilacinum strains were prepared as described above. The spores were counted using a hemocytometer, and homogenous suspensions of $10^{3}-10^{4}$ spores $/ \mathrm{mL}$ were prepared.

The MICs of PHMB and four antimicrobial agents, namely methylparaben (FUJIFILM Wako Pure Chemical Co., Osaka, Japan), phenoxyethanol (FUJIFILM Wako Pure Chemical Co., Osaka, Japan), benzisothiazolin-3-one (Lonza, Basel, Switzerland), and benzalkonium chloride (Kao Co., Tokyo, Japan) 

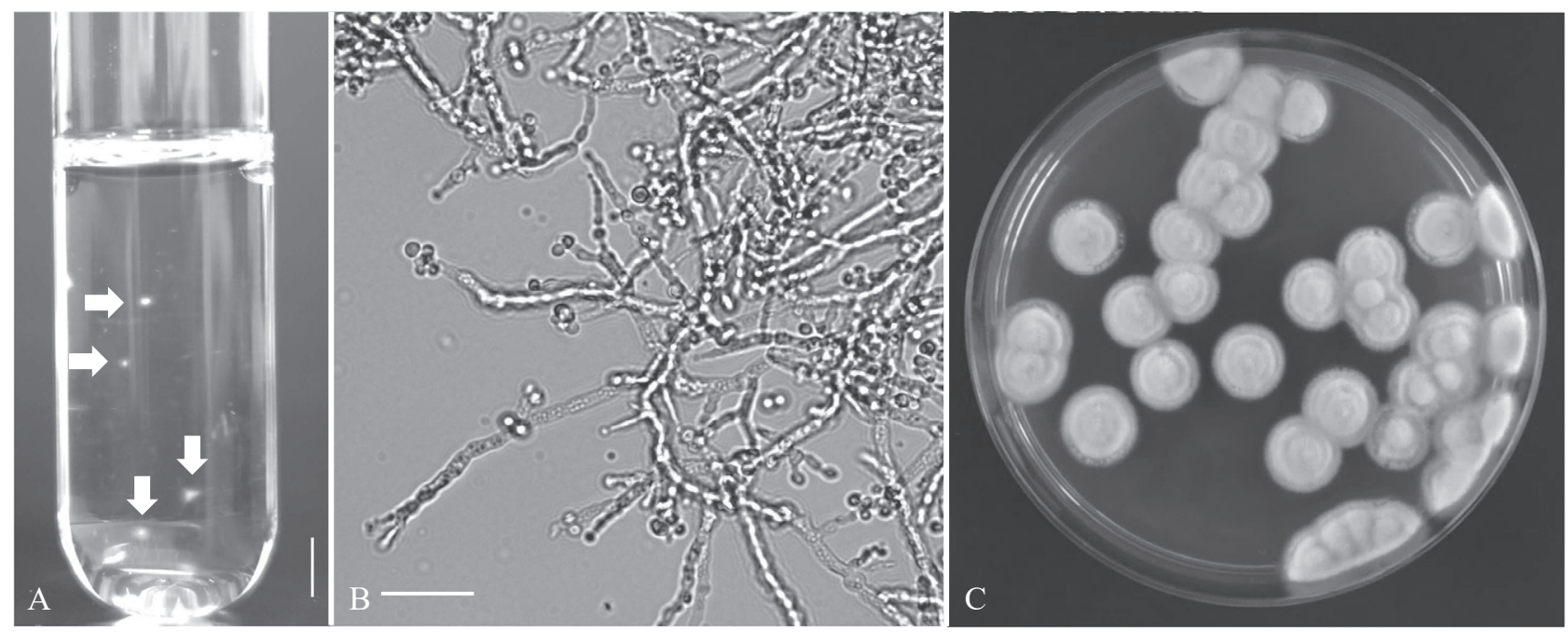

FIG. 1. (A) White floating masses in a $20 \%$ aqueous solution of polyhexamethylene biguanide hydrochloride (PHMB). Scale bar $=5 \mathrm{~mm}$. White arrows indicate the floating masses that were observed after leaving the PHMB in polyethylene containers to stand for more than 1 month. (B) Mycelia and spores of the white floating masses observed in a 20\% solution of PHMB. Scale bar $=20 \mu \mathrm{m}$ (light microscope). (C) Colonies formed from mycelium of the floating masses inoculated on potato dextrose agar at $22.5^{\circ} \mathrm{C}$ for 6 days.

against $P$. lilacinum strains and other fungi were determined using the broth dilution method (Wiegand et al., 2008), with minor modifications. Briefly, $0.1 \mathrm{~mL}$ of spore suspension containing $10^{3}$ spores $/ \mathrm{mL}$ and 0.1 $\mathrm{mL} 5$-fold diluted potato dextrose broth containing various concentrations of antimicrobial agents were mixed well in 96-well microtiter plates (AGC Techno Glass CO., LTD., Shizuoka, Japan). The final concentrations were $100-100,000 \mu \mathrm{g} / \mathrm{mL}, 2-1,000 \mu \mathrm{g} / \mathrm{mL}, 21-11,000$ $\mu \mathrm{g} / \mathrm{mL}, 1.6-1,700 \mu \mathrm{g} / \mathrm{mL}$, and $2.4-2,500 \mu \mathrm{g} / \mathrm{mL}$ for PHMB, methylparaben, phenoxyethanol, benzisothiazolin-3-one, and benzalkonium chloride, respectively, in serial two-fold dilutions. Duplicate mixtures were incubated at $25^{\circ} \mathrm{C}$ for 3 days to determine the MICs.

The MICs of seven antifungal agents including micafungin, amphotericin B, 5-flucytosine, fluconazole, itraconazole, voriconazole, and miconazole for the type and resistant strains were determined by the microdilution method for filamentous fungi as proposed by the Clinical and Laboratory Standards Institute (document M38-A2, 2008). Spore suspensions of $10^{4}$ spores $/ \mathrm{mL}$ were prepared in RPMl 1640 broth and transferred to microtiter plates of an antifungal sensitivity kit (Dry Plate; Eiken Chemicals Co., Ltd., Tokyo, Japan), which were incubated at $35^{\circ} \mathrm{C}$. After $48 \mathrm{~h}$, growth inhibition was examined visually.

\section{Genome sequencing, assembly, and annotation}

The genomic DNA of the fungal isolate was extracted from a one-day-old culture using phenol-chloroform extraction and Nucleobond AXG column (Takara) with Nucleobond buffer set III (Takara). Genome sequencing was performed on a Pacific Biosciences RS II (Pacific Biosciences, Menlo Park, CA, USA) using libraries prepared with the SMRT- bell template prep kit 1.0 (Pacific Biosciences). A draft genome of the fungal isolate was assembled using SMRT Analysis 2.3 (Pacific Biosciences). The sequencing runs and assembly of the libraries were conducted by Takara Bio (Mie, Japan).

Genome annotation was performed by using the Funannotate (v.1.7.4) pipeline (Palmer, 2016). The soft-masked genome by RepeatMasker (v.4.0.7) (Smit et al., 2018) and RepeatModeler (v.1.0.11) (Smit and Hubley, 2008) was used for generating an ab initio gene model with the options --busco_seed_species "fusarium_ graminearum" and --busco_db "sordariomycetes". The completeness of the assembly and gene prediction was assessed using BUSCO v.4.0.5 with 3817 Sordariomycetes ortholog genes (Seppey et al., 2019).

\section{RESULTS}

\section{Isolation of fungi from $\mathbf{2 0} \%$ aqueous solution of PHMB}

White floating masses in a $20 \%$ aqueous solution of PHMB were collected for identification (FIG. 1A). The masses were first observed under a light microscope, which revealed the presence of fungal mycelial networks and spores (FIG. 1B). Pieces of mycelial structures were inoculated on PDA plates and incubated at $22.5^{\circ} \mathrm{C}$ for 6 days. After cultivation, numerous brownish colonies 


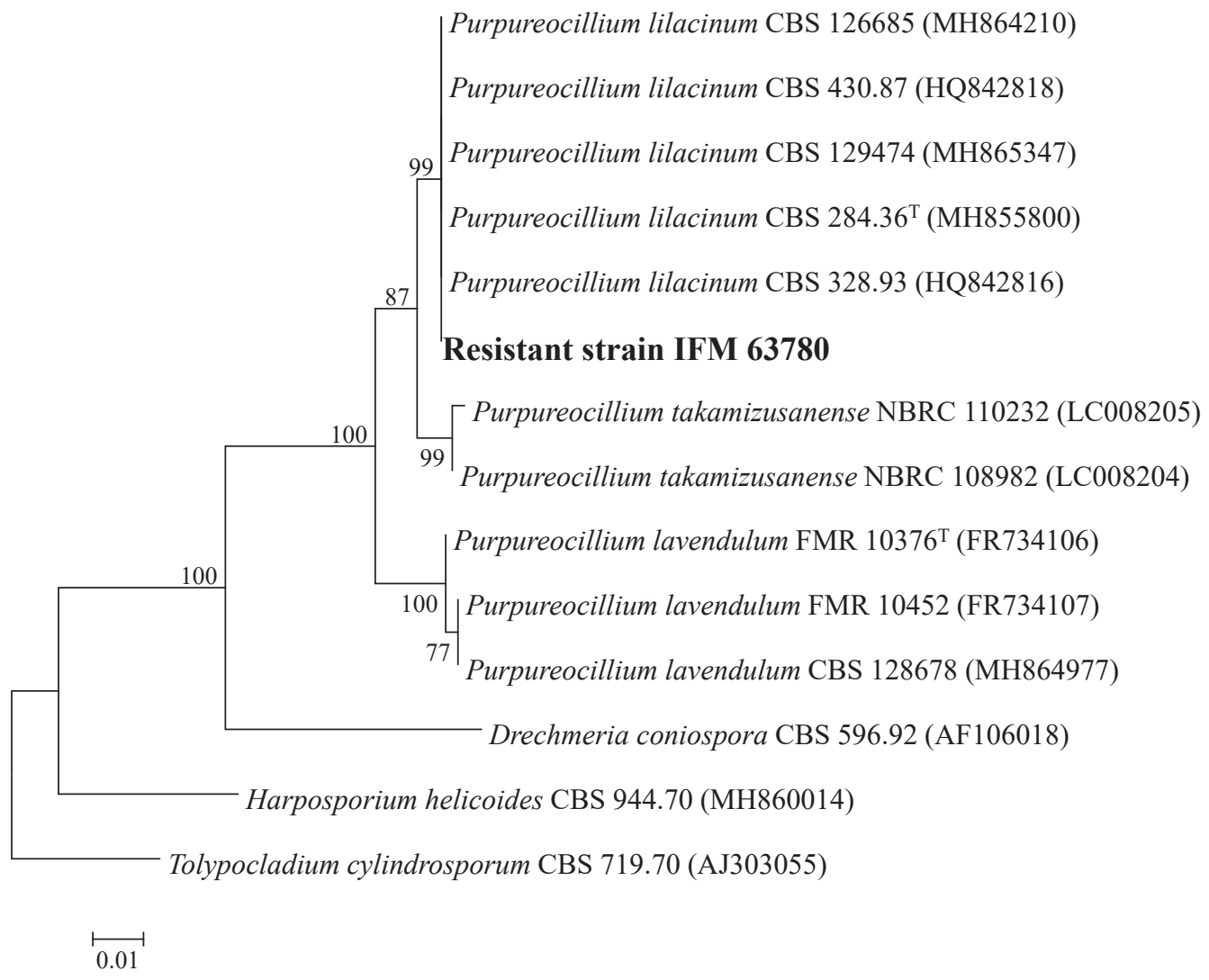

FIG. 2. Neighbor-joining tree of the polyhexamethylene biguanide hydrochloride-resistant strain and its relatives based on ITS region sequences. Bootstrap values above $70 \%$ are indicated near each cluster; scale bar at the bottom left indicates the number of substitutions per site; T: ex-type strain of the species; ITS: internal transcribed spacer.

were formed on all PDA plates (FIG. 1C).

\section{Identification of fungal isolates}

Among the numerous brownish colonies isolated on PDA plates, five colonies were randomly picked for microbial identification based on ITS sequencing. The ITS sequences of the isolates were identical, and phylogenetic analysis revealed that they were highly similar (98.1-100\%) to that of P. lilacinum (FIG. 2). Based on their morphology and ITS-based phylogeny, the isolates were all identified as $P$. lilacinum. One of the five strains, termed IFM 63780, was used in subsequent experiments as a representative PHMB-resistant fungal isolate.

\section{Morphology of the type and resistant strain}

The morphological characteristics of the PHMBresistant strain IFM 63780 were compared with those of the type strain IFM $47467^{\top}$. IFM 63780 colonies on PDA were slightly wrinkled and brownish like the type strain, and their growth was slower than that of the type strain (FIG. 3). Based on SEM, the conidiospore morphology and hyphal formation of IFM 63780 were similar to those of IFM $47467^{\top}$ (FIG. 4). These results indicated no significant difference in morphology and growth between the type strain IFM $47467^{\top}$ and the resistant strain IFM 63780.

\section{PHMB susceptibility of $\boldsymbol{P}$. lilacinum strains}

The PHMB susceptibility of seven cultured $P$. lilacinum strains and the resistant strain IFM 63780 was investigated by counting live spores after inoculating spore suspensions in freshly prepared $20 \%$ aqueous solution of PHMB. P. lilacinum IFM $47467^{\top}$ spores were killed within 3 days, and the live spore counts for all other strains were decreased below the detection limit in 14 to 28 days (TABLE 2). In contrast, the live spore count of IFM 63780 did not decrease over 28 days, and white floating masses were observed within 7 days after inoculation and were confirmed by light microscopy to consist of mycelia. These results indicated that PHMB susceptibility of IFM 63780 is extremely low and that this strain can proliferate in a highly concentrated PHMB solution. A similar proliferative potential of IFM 63780 in a $20 \%$ aqueous solution of PHMB was observed after the fifth 

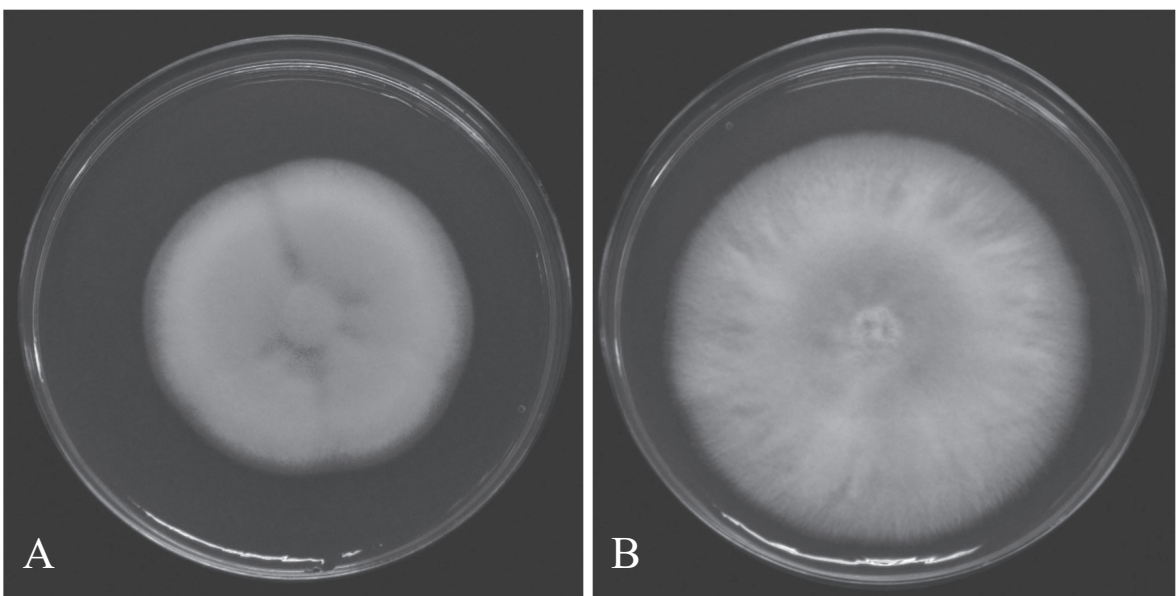

FIG. 3. Purpureocillium lilacinum colonies on potato dextrose agar after incubation at $25^{\circ} \mathrm{C}$ for 14 days. IFM 63780 (A) IFM $47467^{\top}$ (B).
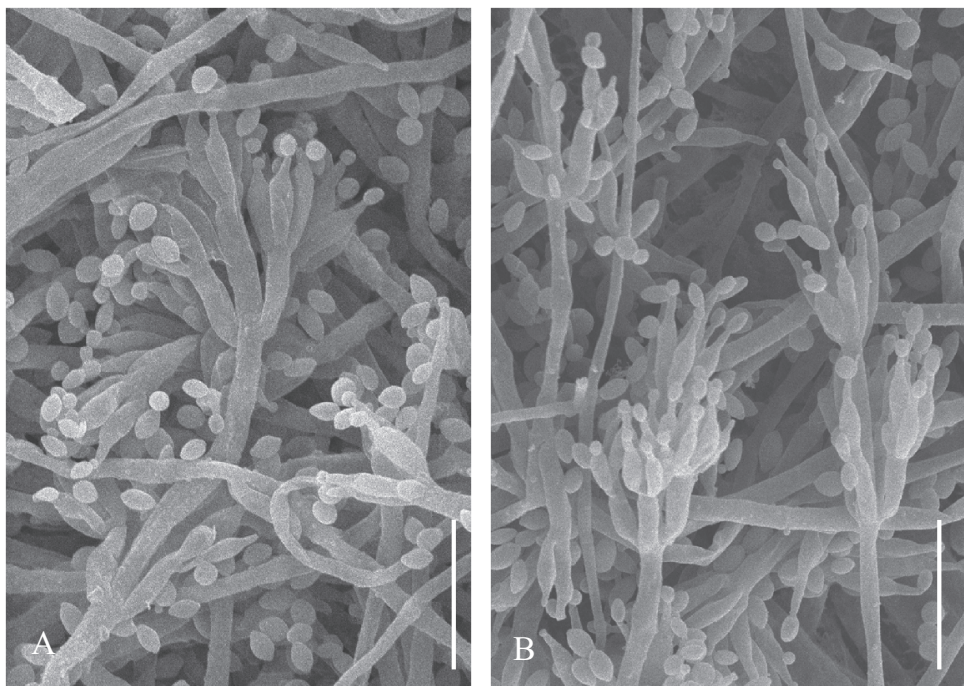

FIG. 4. Scanning electron micrographs of Purpureocillium lilacinum grown on potato dextrose agar at $25^{\circ} \mathrm{C}$ for 14 days. IFM 63780 (A), IFM $47467^{\top}$ (B). Scale bar $=10 \mu \mathrm{m}$.

TABLE 2. Purpureocillium lilacinum survival (log CFU/mL) in $20 \%$ aqueous solution of PHMB

\begin{tabular}{lccccc}
\hline \multicolumn{1}{c}{ Strains } & 0 & 3 days & 7 days & 14 days & 28 days \\
\hline IFM 63780 & 4.0 & 4.0 & $>4.0$ & $>4.0$ & $>4.0$ \\
IFM 47467 & 4.0 & $<2.0$ & $<2.0$ & $<2.0$ & $<2.0$ \\
NBRC 5752 & 4.0 & 3.9 & 3.5 & 2.6 & $<2.0$ \\
NBRC 31847 & 4.0 & 4.0 & 4.0 & 3.5 & $<2.0$ \\
NBRC 31914 & 4.0 & 3.4 & 3.3 & $<2.0$ & $<2.0$ \\
NBRC 31970 & 4.0 & 3.5 & 2.8 & $<2.0$ & $<2.0$ \\
NBRC 32861 & 4.0 & 4.0 & 3.9 & 3.4 & $<2.0$ \\
NBRC 103232 & 4.0 & 3.7 & 3.0 & 2.3 & $<2.0$ \\
\hline
\end{tabular}

$\mathrm{CFU}$, colony forming units; PHMB, Polyhexamethylene biguanide hydrochloride 
passage on PDA (data not shown), indicating strong and stable $\mathrm{PHMB}$ resistance of the isolate.

\section{Comparison of MICs}

The MICs of PHMB, four major antimicrobial agents, and seven antifungal agents for IFM 63780 in comparison with IFM $47467^{\top}$, determined by the broth dilution method, are shown in TABLES 3 and 4, respectively. The MIC of PHMB for IFM 63780 was 50,000 $\mu \mathrm{g} / \mathrm{mL}$, which was approximately four-fold the MIC for IFM $47467^{\top}$ (TABLE 3). Unexpectedly, the MICs of methylparaben, phenoxyethanol, benzisothiazolin-3-one, and benzalkonium chloride for IFM 63780 were lower than or equal to those against IFM $47467^{\top}$. A similar trend was observed for the antifungal agents, micafungin, amphotericin B, 5-flucytosine, fluconazole, itraconazole, voriconazole, and miconazole (TABLE 4). These results indicated that IFM 63780 is less susceptible than IFM $47467^{\top}$ only to PHMB. The MICs of PHMB in four other pathogenic filamentous fungi and two yeasts were below $400 \mu \mathrm{g} / \mathrm{mL}$ and were consistently low (TABLE 5). Together, these results showed that $P$. lilacinum is significantly resistant to $\mathrm{PHMB}$, with the specific isolate discussed in this study showing extreme resistance.

\section{Genomic features of the PHMB-resistant strain}

We obtained a high-quality draft genome of IFM 63780 strain, comprising 11 scaffolds with a total length of $37,957,381 \mathrm{bp}$, an $N_{50}$ value of 4,556,994 bases, and a GC content of $58.5 \%$. The completeness of the draft genome was $99.8 \%$ with BUSCO. We predicted a total of 10,540 protein-coding genes. We found no gene homologous to NCW2 that is responsible for cell tolerance to PHMB in yeasts after searching for genes previously reported to be involved in PHMB tolerance in S. cerevisiae (Elsztein et al., 2016; Queiroz et al., 2020).

\section{DISCUSSION}

Purpureocillium lilacinum, formerly known as Paecilomyces lilacinus, is a ubiquitous and saprobic filamentous fungus commonly isolated from soil, decaying vegetation, insects, and nematodes (Guo et al., 2019). P. lilacinum is also a pathogen that causes hyalohyphomycosis in humans (Peixoto et al., 2014), and reportedly produces paecilotoxin (leucinostatin) (Mikami et al., 1989). In this study, we successfully isolated a fungus from a highly concentrated (20\%) PHMB aqueous solution and identified the PHMBresistant isolates as $P$. lilacinum. Previously, Huang et al. (2017) reported $100 \mu \mathrm{g} / \mathrm{mL}$ PHMB-tolerant clinical isolates of Acanthamoeba castellanii (Huang et al., 2017). However, resistance to the extremely high PHMB concentration of $20 \%$, which is $500-10,000$-fold higher than that of industrially recommended concentrations, has not been reported to date. Such highly PHMBresistant $P$. lilacinum strains can cause spoilage and contaminate numerous PHMB-containing products, such as medical supplies, cosmetics, and household products, as well as production lines and public places (e.g., pools and bathing houses) where PHMB is used for decontamination. PHMB also serves as a preservative in solutions for contact lenses; thus, the resistant strains increase the risk of infections, particularly endophthalmitis (Guo et al., 2019), keratitis (Almeida et al., 2019), and skin infections (Trinh and Angarone, 2017). Therefore, our findings have considerable implications for microbial control. Indeed, our characterization of this pathogenic, highly drug-resistant fungus could be valuable for manufacturers and consumers of PHMB-related products, aid in developing approaches to contain/treat contaminations, and prevent further emergence of drug resistance in fungi.

With regard to the microbiological profile of the new isolate, IFM 63780 could even proliferate in a 20\% aqueous solution of $\mathrm{PHMB}$, indicating that $\mathrm{PHMB}$ has no fungistatic or fungicidal effect on this strain. It is plausible that this isolate utilizes PHMB as a sole carbon source. Our study revealed no significant difference in terms of morphology and growth between the type strain IFM $47467^{\top}$ and the resistant strain IFM 63780. Moreover, no unusual cell structures such as thick-walled chlamydospores or hyphae, which are responsible for heat resistance in Paecilomyces variotii (Piecková and Samson, 2000), a typical heat-resistant fungus, were observed. The high resistance of IFM 63780 may be related to hitherto unidentified molecular mechanisms other than structural rearrangements of the cell membrane.

P. linacinum is resistant to PHMB as a species-level, and among them, the IFM63780 isolated this time exhibited extremely high resistance. MIC value for IFM 63780 was $50,000 \mu \mathrm{g} / \mathrm{mL}$, which was significantly higher than the concentrations for the manufacturer's recommendation $(20-400 \mu \mathrm{g} / \mathrm{mL})$. As PHMB is a cationic polymer, we expected strain IFM 63780 to be highly resistant to cationic agents; however, the MIC of benzalkonium chloride for IFM 63780 was identical to that for IFM $47467^{\top}$. P. lilacinum is known to be sensitive to voriconazole, but resistant to other antifungal agents, including amphotericin B (Luangsa-Ard et al., 2011), and the antifungal susceptibility test results for IFM 63780 were consistent with previous findings. IFM 63780 showed particularly high resistance, specifically to PHMB. Multidrug resistance refers to simultaneous resistance to unrelated drugs (Alekshun and Levy, 2007). It has been recommended that combinations of 
TABLE 3. MIC of PHMB and other antimicrobial agents for IFM 63780 and IFM $47467^{\top}$

\begin{tabular}{lcc}
\hline \multicolumn{2}{c}{$\mathrm{MIC}(\mu \mathrm{g} / \mathrm{mL})$} \\
\hline Antimicrobial agent & $\begin{array}{c}\text { IFM 63780 } \\
\left(10^{3} \text { spores } / \mathrm{mL}\right)\end{array}$ & $\begin{array}{c}\mathrm{IFM} 47467^{\top} \\
\left(10^{3} \text { spores } / \mathrm{mL}\right)\end{array}$ \\
\hline PHMB & 50,000 & 13,000 \\
Methylparaben & 250 & 500 \\
Phenoxyethanol & 2,800 & 5,500 \\
Benzisothiazolin-3-one & $<1.6$ & 3.2 \\
Benzalkonium chloride & 20 & 20 \\
\hline
\end{tabular}

MIC, minimum inhibitory concentration; PHMB, Polyhexamethylene biguanide hydrochloride

TABLE 4. MIC of antifungal agents for IFM 63780 and IFM $47467^{\top}$

\begin{tabular}{|c|c|c|}
\hline \multirow[b]{2}{*}{ Antifungal agent } & \multicolumn{2}{|c|}{ MIC $(\mu \mathrm{g} / \mathrm{mL})$} \\
\hline & $\begin{array}{c}\text { IFM } 63780 \\
\left(10^{4} \text { spores } / \mathrm{mL}\right)\end{array}$ & $\begin{array}{c}\text { IFM } 47467^{\top} \\
\left(10^{4} \text { spores } / \mathrm{mL}\right)\end{array}$ \\
\hline Micafungin (MEC) & $<0.015$ & 0.03 \\
\hline Amphotericin B & $>16$ & $>16$ \\
\hline 5-Flucytosine & $>64$ & $>64$ \\
\hline Fluconazole & 16 & 16 \\
\hline Itraconazole & 2.0 & 2.0 \\
\hline Voriconazole & 0.25 & 0.25 \\
\hline Miconazole & 2.0 & 2.0 \\
\hline
\end{tabular}

MIC, minimum inhibitory concentration

MEC, minimum effective concentration

TABLE 5. MICs of PHMB for other pathogenic filamentous fungi and yeasts

\begin{tabular}{lc}
\hline \multicolumn{1}{c}{$\begin{array}{c}\text { Inoculum } \\
\left(10^{3} \text { spores/mL) }\right.\end{array}$} & MIC $(\mu \mathrm{g} / \mathrm{mL})$ \\
\hline Aspergillus fumigatus IFM 54229 & $<400$ \\
Aspergillus niger IFM 62238 & $<400$ \\
Fusarium solani IFM 62065 & $<400$ \\
Penicillium citrinum IFM 62178 & $<400$ \\
Candida albicans IFM 40009 & $<400$ \\
Cryptococcus neoformans IFM 46660 & $<400$ \\
\hline
\end{tabular}

MIC, minimum inhibitory concentration; PHMB, Polyhexamethylene biguanide hydrochloride

multiple antimicrobial agents should be used to prevent the emergence of resistance to a single antimicrobial agent. Therefore, the combined antimicrobial spectrum would be extended, and the required concentration would decrease (Ettayebi et al., 2000; Leekha et al., 2011). With regard to the PHMB-resistant strain isolated in this study, multidrug resistance was not clearly observed; however, the possibility of acquiring multidrug resistance is conceivable based on previously reported cases.

IFM 63780 genome size, GC content, and gene count are almost identical to those of other $P$. lilacinus strains, such as PLFJ-1 (38.5 Mb, 58.3\%, 11,763 genes, respectively) (Wang et al., 2016) and 36-1 (37.6 Mb, 
58.4\%, 13,150 genes, respectively) (Xie et al., 2016). Although we expected a similar resistance mechanism and intended to identify a gene similar to that previously reported in yeast, no gene homologous to NCW2 of S. cerevisiae was found in the genome of IFM 63780. This result suggests that IFM 63780 acquired and developed $\mathrm{PHMB}$ resistance through a mechanism that differs from that of yeasts and potentially involves PHMB resistance-related genes that are unique to filamentous fungi. The availability of the IFM 63780 genome sequence will contribute to our understanding of the molecular mechanisms underlying the biology of $P$. lilacinus and other fungi.

Our results justify efforts to increase manufacturer and consumer awareness regarding the use of PHMB. Additional studies are required to elucidate the resistance gene(s) and mechanism(s) of PHMB in the genus Purpureocillium and devise strategies to prevent the emergence of such highly drug-resistant fungi.

In conclusion, we identified a fungus contaminating a $20 \%$ aqueous solution of PHMB as P. lilacinum. The resistance level of the isolate, IFM 63780, was markedly higher than that of previously reported PHMB-resistant microorganisms. Moreover, the strain was found to be specifically resistant to PHMB among all antimicrobial agents tested in this study; however, its genome possessed no gene homologous to the PHMB resistance-related gene in yeasts. Our findings highlight the medical implications and public health risks pertaining to the emergence of drug resistance microorganisms and indicate the presence of a novel drug resistance mechanism operating in the genus Purpureocillium.

\section{ACKNOWLEDGMENT}

\author{
Not applicable
}

\section{REFERENCES}

Alekshun, M. N., and Levy, S. B. (2007) Molecular mechanisms of antibacterial multidrug resistance. Cell, 128, 1037-1050.

Allen, M. J., White, G. F., and Morby, A. P. (2006) The response of Escherichia coli to exposure to the biocide polyhexamethylene biguanide. Microbiology (Reading)., 152, 989-1000.

Almeida Oliveira, M., Carmo, A., Rosa, A., and Murta, J. (2019) Posaconazole in the treatment of refractory Purpureocillium lilacinum (former Paecilomyces lilacinus) keratitis: the salvation when nothing works. BMJ Case Rep., 12, e228645.

Arch Chemicals, Inc., Norwalk, CT, USA, VANTOCIL ${ }^{\mathrm{TM}} \mathrm{IB}$ technical information bulletin, http://www.excelind.co.in/ Excel_Chemical/download/Vantocil_IB_Tech.pdf; 2005. Accessed 16 Dec 2019.

Chadeau, E., Brunon, C., Degraeve, P., Léonard, D., Grossiord, C., Bessueille, F., Cottaz, A., Renaud, F., Ferreira, I., Darroux, C., Simon, F, Rimbault, F., and Oulahal, N.
(2012) Evaluation of antimicrobial activity of a polyhexamethylene biguanide-coated textile by monitoring both bacterial growth (iso 20743/2005 standard) and viability (live/dead baclight kit). J. Food Saf., 32, 141-151.

Clinical and Laboratory Standards Institute Reference method for broth dilution antifungal susceptibility testing of filamentous fungi; approved standard-2nd ed, CLSI document M38-A2, 2008. Clinical and Laboratory Standards Institute, Wayne, PA.

Elsztein, C., de Cassia de Lima, R., de Barros Pita, W., and de Morais, M. A. Jr. (2016) NCW2, a gene involved in the tolerance to polyhexamethylene biguanide (PHMB), may help in the organisation of $\beta-1,3$-glucan structure of Saccharomyces cerevisiae cell wall. Curr. Microbiol., 73, 341-345.

Elsztein, C., de Lucena, R. M., and de Morais, M. A. Jr. (2011) The resistance of the yeast Saccharomyces cerevisiae to the biocide polyhexamethylene biguanide: involvement of cell wall integrity pathway and emerging role for YAP1. BMC Mol. Biol., 12, 38.

Ettayebi, K., El Yamani, J., and Rossi-Hassani, B. (2000) Synergistic effects of nisin and thymol on antimicrobial activities in Listeria monocytogenes and Bacillus subtilis. FEMS Microbiol. Lett., 183, 191-195.

Felsenstein, J. (1985) Confidence limits on phylogenies: an approach using the bootstrap. Evolution, 39, 783-791.

Feng, L., Wu, F., Li, J., Jiang, Y., and Duan, X. (2011) Antifungal activities of polyhexamethylene biguanide and polyhexamethylene guanide against the citrus sour rot pathogen Geotrichum citri-aurantii in vitro and in vivo. Postharvest Biol. Technol., 61, 160-164.

Guo, L. N., Wang, H., Hsueh, P. R., Meis, J. F., Chen, H., and Xu, Y. C. (2019) Endophthalmitis caused by Purpureocillium lilacinum. J. Microbiol. Immunol. Infect., 52, 170-171.

Henly, E. L., Dowling, J. A. R., Maingay, J. B., Lacey, M. M., Smith, T. J., and Forbes, S. (2019) Biocide exposure induces changes in susceptibility, pathogenicity, and biofilm formation in uropathogenic Escherichia coli. Antimicrob. Agents Chemother., 63, e01892-18.

Huang, F. C., Shih, M. H., Chang, K. F., Huang, J. M., Shin, J. W., and Lin, W. C. (2017) Characterizing clinical isolates of Acanthamoeba castellanii with high resistance to polyhexamethylene biguanide in Taiwan. J. Microbiol. Immunol. Infect., 50, 570-577.

Hubner, N. O., and Kramer, A. (2010) Review on the efficacy, safety and clinical applications of polihexanide, a modern wound antiseptic. Skin Pharmacol. Physiol., 23, 17-27.

Kimura, M. (1980) A simple method for estimating evolutionary rates of base substitutions through comparative studies of nucleotide sequences. J. Mol. Evol., 16, 111-120.

Leekha, S., Terrell, C. L., and Edson, R. S. (2011) General principles of antimicrobial therapy. Mayo Clin. Proc., 86, 156-167.

Luangsa-Ard, J., Houbraken, J., van Doorn, T., Hong, S. B., Borman, A. M., Hywel-Jones, N. L., and Samson, R. A. (2011) Purpureocillium, a new genus for the medically important Paecilomyces lilacinus. FEMS Microbiol. Lett., 321, 141-149.

Makela, P. M., Korkeala, H. J., and Sand, E. K. (1991) Effectiveness of commercial germicide products against the ropy slime-producing lactic acid bacteria. J. Food Prot., 54, 632-636.

Marmur, J. (1961) A procedure for the isolation of deoxyribonucleic acid from microorganisms. J. Mol. Bio., 3, 208-218.

Mashat, B. H. (2016) Polyhexamethylene biguanide 
hydrochloride: features and applications. British Journal of Environmental Science, 4, 49-55. ISSN 2055-0219(Print), ISSN 2055-0227 (online)

Messick, C. R., Pendland, S. L., Moshirfar, M., Fiscella, R. G., Losnedahl, K. J., Schriever, C. A., and Schreckenberger, P. C. (1999) In-vitro activity of polyhexamethylene biguanide (PHMB) against fungal isolates associated with infective keratitis. J. Antimicrob. Chemother., 44, 297-298.

Mikami, Y., Yazawa, K., Fukushima, K., Arai, T., Udagawa, S., and Samson, R. A. (1989) Paecilotoxin production in clinical or terrestrial isolates of Paecilomyces lilacinus strains. Mycopathologia, 108, 195-199.

O'Donnell, K. (1993) Fusarium and its near relatives. In The fungal holomarph, mititic, meiotic and pleomorpic speciation in fungal systematic (Reynolds, D. R., and Taylor, J. W., eds.), pp. 225-233, CAB International, Wallingford.

Palmer, J. (2016) Funannotate: pipeline for genome annotation. https://funannotate.readthedocs.io/en/latest/index. html. Accessed 2 Mar 2020.

Peixoto, M. L. P., Santos, D. O., de Castro Campos de Souza, I., Neri, E. C. L., de Sequeira, D. C. M., De Luca, P. M., and de Moraes Borba, C. (2014) Interaction of an opportunistic fungus Purpureocillium lilacinum with human macrophages and dendritic cells. Rev. Soc. Bras. Med Trop., 47, 613-617.

Piecková, E., and Samson, R. A. (2000) Heat resistance of Paecilomyces variotii in sauce and juice. J. Ind. Microbiol. Biotechnol., 24, 227-230.

Queiroz, M. G., Elsztein, C., and de Morais, M. A. Jr. (2020) The effects of the Ncw2 protein of Saccharomyces cerevisiae on the positioning of chitin in response to cell wall damage. Antonie van Leeuwenhoek., 113, 265-277.

Saitou, N., and Nei, M. (1987) The neighbor-joining method: a new method for reconstructing phylogenetic trees. Mol. Biol. Evol., 4, 406-425.

Seppey, M., Manni, M., and Zdobnov, E. M. (2019) BUSCO: assessing genome assembly and annotation completeness. Methods Mol. Biol., 1962, 227-245.

Smit, A. F. A., and Hubley, R. (2008) RepeatModeler Open1.0. http://www.repeatmasker.org. Accessed 2 Mar 2020.

Smit, A. F. A., Hubley, R., and Green, P. (2018)
RepeatMasker Open-4.0. http://www.repeatmasker.org. Accessed 2 Mar 2020.

Tamura, K., Stecher, G., Peterson, D., Filipski, A., and Kumar, S. (2013) MEGA6: Molecular Evolutionary Genetics Analysis version 6.0. Mol. Biol. Evol., 30, 2725-2729.

Thompson, J. D., Higgins, D. G., and Gibson, T. J. (1994) CLUSTAL W: improving the sensitivity of progressive multiple sequence alignment through sequence weighting, positionspecific gap penalties and weight matrix choice. Nucleic Acids Res., 22, 4673-4680.

Thomson, J. E., Cox, N. A., Bailey, J. S., and Islam, M. N. (1981) Minimizing salmonella contamination on broiler carcasses with poly (hexamethylenebiguanide hydrochloride). J. Food Prot., 44, 440-441.

Trinh, S. A., and Angarone, M. P. (2017) Purpureocillium lilacinum tattoo-related skin infection in a kidney transplant recipient. Transpl. Infect. Dis., 19, e12689.

Valluri, S., Fleming, T. P., Laycock, K. A., Tarle, I. S., Goldberg, M. A., Garcia-Ferrer, F. J., Essary, L. R., and Pepose, J. S. (1997) In vitro and in vivo effects of polyhexamethylene biguanide against herpes simplex virus infection. Cornea., 16, 556-559.

Wang, G., Liu, Z., Lin, R., Li, E., Mao, Z., Ling, J., Yang, Y., Yin, W. B., and Xie, B. (2016) Biosynthesis of antibiotic leucinostatins in bio-control fungus Purpureocillium lilacinum and their inhibition on phytophthora revealed by genome mining. PLoS Pathog., 12, e1005685.

White, T. J., Bruns, T., Lee, S., and Taylor, J. (1990) Amplification and direct sequencing of fungal ribosomal RNA Genes for phylogenetics. In PCR protocols, a guide to methods and applications (Innis, M. A., Gelfand, D. H., Sninsky, J. J., and White, T. J., eds.), pp. 315-322, Academic Press, San Diego.

Wiegand, I., Hilpert, K., and Hancock, R. E. W. (2008) Agar and broth dilution methods to determine the minimal inhibitory concentration (MIC) of antimicrobial substances. Nat. Protoc., 3, 163-175.

Xie, J., Li, S., Mo, C., Xiao, X., Peng, D., Wang, G., and Xiao, Y. (2016) Genome and transcriptome sequences reveal the specific parasitism of the nematophagous Purpureocillium lilacinum 36-1. Front Microbiol., 7, 1084. 